SHORT REPORT

\title{
Sleep disorders and their determinants in multiple system atrophy
}

\author{
I Ghorayeb, F Yekhlef, V Chrysostome, E Balestre, B Bioulac, F Tison
}

J Neurol Neurosurg Psychiatry 2002;72:798-800

Objectives: To evaluate the incidence, types, determinants, and consequences of sleep disorders in patients with multiple system atrophy (MSA) and determine whether their characteristics are similar to those of patients with Parkinson's disease (PD).

Methods: Information about sleep disorders was collected using a standardised questionnaire in an unselected group of 57 patients with MSA and in 62 patients with PD matched as a group for age, sex distribution, and disease duration.

Results: Seventy percent of patients with MSA complained of sleep disorders compared with $51 \%$ of patients with PD $(p=0.03)$. The most commonly reported sleep disorders were sleep fragmentation $(52.5 \%)$, vocalisation $(60 \%)$, REM sleep behaviour disorder (47.5\%), and nocturnal stridor (19\%). Except for sleep fragmentation, the incidence of these disorders was significantly higher than in PD. Sleep problems tended to be associated with more severe motor symptoms, longer disease duration, depression, and longer duration of levodopa treatment. Half of patients with MSA with sleep disorders complained of daytime somnolence compared with $30 \%$ of patients with PD. Daytime somnolence was significantly associated with disease severity in MSA.

Conclusion: This study shows that sleep disorders are more common in patients with MSA than in those with PD after the same duration of the disease, reflecting the more diffuse underlying pathological process in MSA.

S eep disorders are common in Parkinson's disease (PD) ${ }^{1-3}$ and multiple system atrophy (MSA), ${ }^{4-6}$ with increasing evidence implicating the underlying pathological process. ${ }^{7-9}$ The latter is more diffuse in MSA and results in a more aggressive course and levodopa unresponsiveness. ${ }^{10}$ Although the two disorders share parkinsonism, little is known about whether sleep disorders during them are similar. A comparison of sleep disorders in the two conditions may prove useful in elucidating their determinants. Only one study has previously compared sleep disturbances in a small group of patients with PD or MSA. ${ }^{6}$ We therefore compared the incidence, characteristics, and determinants of sleep problems in a sample of 57 patients with MSA and 62 matched patients with PD.

\section{PATIENTS AND METHODS}

The University of Bordeaux 2 ethical committee approved the study. Fifty seven consecutive patients with MSA underwent a standardised face to face interview and examination protocol to fulfil MSA clinical diagnostic criteria for "possible" and "probable" MSA. ${ }^{11}$ Comparison was made with 62 outpatients with PD, who were recruited in parallel and matched as a group for sex, age, and disease duration.
Semiquantitative scales rating parkinsonism severity (Hoehn and Yahr stage, unified Parkinson's disease rating scale (UPDRS-III) motor score, Schwab and England activity of daily living scale), self reported depressive symptoms (Center for Epidemiological Studies depression scale, CES-D), and mini mental state examination (MMSE) were administered. A structured interview collected data on disease history and medication. The standardised interview also assessed sleep problems ("yes" or "no" type of answer). If "yes", they had to specify whether they had: 1 , persistent initial insomnia (difficulty falling asleep within 30 minutes or more); 2, sleep fragmentation (more than two $\geqslant 30$ minute nocturnal awakenings independently of the need to use the bathroom); 3, early awakening (undesirable early morning awakening with inability to fall asleep again); 4, daytime somnolence (undesirable and inappropriate dozing during waking hours). Data were collected with the assistance of the patient's bed partner or an immediate household member for all the patients. Information was also sought on whether patients snored or had nocturnal motor symptoms (pain, cramps, nocturnal involuntary twisting movements, uncontrolled sudden jerks, and other abnormal nocturnal limb movements). Nocturnal stridor, described as a harsh or strained high pitched sound, and talking were investigated from the reports of the patients' bed partners' or caregivers'. Restless legs syndrome and rapid eye movement (REM) sleep behaviour disorder (RBD) were diagnosed according to the International classification of sleep disorders (ICSD) minimal diagnostic criteria. $^{12}$ A Fisher's exact test or a $\chi^{2}$ test was used when appropriate to compare the incidence of sleep disorders, types, determinants, and sex distribution in patients with MSA or PD. The mean age, disease duration and severity, duration of levodopa treatment, daily dose of levodopa, use of dopamine agonists and amantadine ("yes" or "no" type of answer), depressive symptoms, disability, and cognitive impairment scores were compared in patients with MSA or PD with and without sleep complaints using Student's $t$ test.

\section{RESULTS}

The mean age (67.3 (SD 8.5) years), sex distribution, and mean disease duration (5.75 (SD 3.36) years) of the 57 patients with MSA did not differ significantly from those of the 62 patients with PD (65.2 (SD 8.7) years and 7 (SD 4.37) years respectively). Thirty percent of patients with MSA were classified as MSA-C and 70\% as MSA-P.

Parkinsonism was significantly more severe in patients with MSA than in those with PD (mean Hoehn and Yahr score $=3.23($ SD 1.36) $v 2.18($ SD 0.76), $\mathrm{p}<0.0001$; mean

Abbreviations: PD, Parkinson's disease; MSA, multiple system atrophy; REM, rapid eye movement; RBD, REM sleep behaviour disorder; UPDRS, unified Parkinson's disease rating scale; MMSE, mini mental state examination 
Table 1 Nighttime problems in patients with Parkinson's disease (PD) and multiple system atrophy (MSA)

\begin{tabular}{|c|c|c|c|c|c|c|c|c|c|}
\hline Patients (no) & SC & Insomnia & SF & EA & Vocalisation & RBD & RLS & Snoring & Stridor \\
\hline PD (62) & 51 & 19 & 38.7 & 42 & 13 & 9.7 & 3.2 & 56.7 & 0 \\
\hline MSA (57) & $70 *$ & 20 & 52.5 & 32.5 & $60 *$ & $47.5^{*}$ & 12.5 & 72.5 & $19 *$ \\
\hline
\end{tabular}

Table 2 Clinical characteristics of patients with Parkinson's disease (PD) or multiple system atrophy (MSA) with and without sleep complaints

\begin{tabular}{|c|c|c|c|c|}
\hline & $\begin{array}{l}\text { PD patients } \\
\text { with sleep } \\
\text { complaints }\end{array}$ & $\begin{array}{l}\text { PD patients } \\
\text { without sleep } \\
\text { complaints }\end{array}$ & $\begin{array}{l}\text { MSA patients } \\
\text { with sleep } \\
\text { complaints }\end{array}$ & $\begin{array}{l}\text { MSA patients } \\
\text { without sleep } \\
\text { complaints }\end{array}$ \\
\hline Percentage & 51 & 49 & $70 *$ & 30 \\
\hline Age (years) & $66.2(7.9)$ & $64.2(9.7)$ & $67.5(7.8)$ & $67(10.2)$ \\
\hline Men $(\%)$ & 58.06 & 60 & 62.5 & 23.53 \\
\hline Disease duration (years) & $8(4.5)$ & $6(4.1)$ & $6.2(3.2)$ & $4.7(3.6)$ \\
\hline Hoehn and Yahr score & $2.34(0.83)$ & $1.98(0.65)$ & $3.32(1.38)$ & $3.03(1.34)$ \\
\hline UPDRS-III score & $31.13(13.11)$ & $29.18(13.87)$ & $50.33(19.64)$ & $43.29(17.17)$ \\
\hline Schwab and England score & 70.32 (22.13) & 75.67 (15.69) & $40.51(24.7)$ & $44.12(26.71)$ \\
\hline MMSE score & $27.26(2.64)$ & $27.7(2.07)$ & $25.78(3.79)$ & $26.53(2.89)$ \\
\hline Autonomic failure (\%) & 41.94 & 27.59 & 95 & 82.35 \\
\hline CES-D score & $14.04(10.37)$ & 11.9 (10.59) & 21.06 (11.39) & $18.71(9.1)$ \\
\hline Antidepressant use (\%) & 35.48 & 17.24 & 42.5 & 52.94 \\
\hline Daily levodopa (mg) & $\begin{array}{l}626.61 \\
(367.24)\end{array}$ & $512.5(349.82)$ & $\begin{array}{l}565.63 \\
(471.48)\end{array}$ & $\begin{array}{l}484.56 \\
(434.22)\end{array}$ \\
\hline $\begin{array}{l}\text { Duration of levodopa therapy } \\
\text { (years) }\end{array}$ & $6.8(4.01)^{* *}$ & 3.99 (3.49) & $4(3.66)$ & $2.71(3.34)$ \\
\hline Dopamine agonist use (\%) & 74.2 ** & 37.8 & $32.5^{*}$ & 47.1 \\
\hline Amantadine use (\%) & 12.9 & 17.24 & 11.8 & 27.5 \\
\hline \multicolumn{5}{|c|}{$\begin{array}{l}\text { Values are presented as mean (SD) or percentages. } \\
{ }^{*} p<0.05 \text { when compared with PD patients with sleep complaints, }{ }^{* *} p<0.05 \text { when compared with PD } \\
\text { patients without sleep complaints. } \\
\text { UPDRS, Unified Parkinson's diseaserating scale; MMSE, mini mental state examination; CES-D, Center for } \\
\text { Epidemiological Studies depression scale. }\end{array}$} \\
\hline
\end{tabular}

UPDRS-III score $=47.33($ SD 18.08) $v 29.65$ (SD 12.85), $\mathrm{p}<0.0001$; mean Schwab and England score $=41.6$ (SD 25.14) $v 73.06$ (SD 19.13), $\mathrm{p}<0.0001$ ). The mean daily levodopa dose was not significantly different in patients with MSA $(752.7$ (SD 362.1) mg) and those with PD (636.8 (SD 337) mg) nor was the mean duration of dopa treatment (4.4 (SD 3.82) years $v 5.8$ (SD 3.97) years respectively). Some $37 \%$ of patients with MSA used dopamine agonists (bromocriptine, ropinirole, pergolide, piribedil, lisuride, apomorphine) $v 57 \%$ of patients with PD $(p=0.02)$, and $23 \%$ of patients with MSA used amantadine $v 15 \%$ of patients with PD.

\section{Incidence and types of sleep problems}

Table 1 shows the percentage of patients reporting nighttime disturbances in each group. A significantly higher percentage of patients with MSA complained of sleep disorders compared with patients with PD $(70 \% v 51 \%, \mathrm{p}=0.03)$. A significantly higher percentage of patients with MSA reported nighttime vocalisation and RBD than patients with PD $(60 \% \vee 13 \%$, $\mathrm{p}<0.0001 ; 47.5 \% \vee 9.7 \%, \mathrm{p}=0.0006$ respectively). Nocturnal stridor was reported only by patients with MSA (19\%, $\mathrm{p}=0.0004$ ).

\section{Determinants of sleep disturbances}

Table 2 shows the clinical characteristics of patients with MSA or PD, with and without sleep complaints. Patients with MSA or PD with sleep complaints tended to have longer disease duration, higher mean motor scores (UPDRS-III and Hoehn and Yahr stage), were more disabled with respect to acitvities of daily life, and reported more depressive symptoms than those without sleep complaints. Both groups of patients with sleep complaints tended to use higher daily levodopa doses, and patients with PD more often used dopamine agonists than those without sleep complaints. Amantadine use was not associated with more common sleep problems in either group. Unlike in patients with $\mathrm{PD}$, duration of dopa treatment in patients with MSA was not significantly associated with sleep disorders.

\section{Consequences of sleep disturbances}

Use of hypnotic or antidepressant drugs was not significantly different in patients with MSA or PD. Half of patients with MSA compared with $30 \%$ of patients with PD admitted daytime somnolence and were significantly more severely disabled than those without daytime somnolence (mean UPDRS-III score $=55.8($ SD 15.6) $v 42.7($ SD 19.4), $\mathrm{p}=0.03$; mean Hoehn and Yahr stage $=3.84($ SD 0.88$) v 2.8$ (SD 1.6), $\mathrm{p}=0.02$ ).

\section{DISCUSSION}

This is the first questionnaire based study of nighttime problems in a large unselected MSA population compared with patients with PD matched for age, sex, and disease duration. Although such studies may be limited by the accuracy of self reporting compared with polysomnographic studies, they make it possible to screen a broader and more representative sample of patients.

Overall, patients with MSA reported significantly more nighttime problems than patients with PD. In agreement with previous studies, ${ }^{12}{ }^{6}$ difficulty in getting to sleep was similar in the two groups, a finding previously suggested to be mostly related to age. ${ }^{23}$ As already noted in patients with $\mathrm{PD}^{2}{ }^{13}$ sleep fragmentation and early awakening were also often reported 
by patients with MSA. The higher incidence of sleep fragmentation in patients with MSA suggests that the disease severity may favour this. Indeed, as for patients with PD, the mean UPDRS-III and Hoehn and Yahr scores for sleep disordered patients with MSA were higher than in patients with MSA without sleep problems.

Nocturnal vocalisation and RBD were more common in MSA, the former possibly being an early manifestation of RBD in patients with MSA. ${ }^{14}$ This may reflect the more extensive brainstem pathology in MSA. ${ }^{5}{ }^{10}$ Nocturnal stridor occurred in $19 \%$ of patients with MSA, a result in keeping with that obtained by polysomnographic studies. ${ }^{5}{ }^{15}$ Snoring also tended to be more common in patients with MSA. None of our patients with PD reported stridor. This suggests that snoring and stridor are not confused by the patients' bed partners. Although of potential interest, body mass index and neck circumference were not assessed, and there was no obvious clinical evidence of a weight bias between the two groups.

The role of dopaminergic agents in sleep disruption remains a matter of debate. ${ }^{313}{ }^{16}$ On the one hand, the efficacy of dopa treatment and dopamine agonists in our patients with PD may partly explain the reduced occurrence of sleep disturbances compared with patients with MSA, but on the other, dopaminergic drugs may disrupt sleep and induce daytime somnolence. ${ }^{2} 3131617$ Accordingly, duration of dopa treatment, but not daily dose, and agonist use were the most significantly associated with sleep disorders in patients with PD but not in those with MSA.

Even if higher depressive symptom scores were associated with sleep problems in both groups of patients, no significant association was found with antidepressant use. However, these variables may be dependent as both depression and antidepressants may cause sleep disorders. ${ }^{18}$

More patients with MSA complained of daytime somnolence than patients with PD, arguing against the exclusive role of age, as proposed by others. ${ }^{19}$ Patients with MSA with daytime somnolence had significantly higher mean UPDRSIII and Hoehn and Yahr scores than those without, suggesting a probable role for degenerative process diffusion.

\section{Conclusion}

After the same duration of the disease, there was a higher incidence and variety of sleep disorders in patients with MSA than in those with PD. This paralleled the increasing severity of motor signs and the wider diffusion of the underlying degenerative process, which involves neuronal substrates for sleep organisation and maintenance, such as brainstem nuclei and/or basal ganglia.

\section{ACKNOWLEDGEMENTS}

This work was supported by a PHRC 1997 grant, Centre Hospitalier Universitaire de Bordeaux and CNRS (fellowship for I G).

\section{Authors' affiliations}

I Ghorayeb, B Bioulac, Service d'Explorations Fonctionnelles du Système Nerveux, Clinique du Sommeil, Hôpital Pellegrin, Place Amélie Raba-Léon, 33076 Bordeaux cedex, France
F Yekhlef, V Chrysostome, F Tison, Service de Neurologie, Hôpital du Haut Lévêque, Avenue de Magellan, 33600 Pessac cedex, France

E Balestre, INSERM U-330 Biostatistiques et Epidémiologie, Université

Bordeaux 2, 146, rue Léo-Saignat, 33076 Bordeaux cedex, France

Correspondence to: Professor F Tison, Service de Neurologie, Hôpital du Haut Lévêque, Avenue de Magellan, 33600 Pessac cedex, France; francois.tison@chu-bordeaux.fr

Received 9 July 2001

In revised form 11 October 2001

Accepted 13 December 2001

\section{REFERENCES}

1 Factor SA, McAlarney T, Sanchez-Ramos JR, et al. Sleep disorders and sleep effect in Parkinson's disease. Mov Disord 1990;5:280-5.

2 Tandberg E, Larsen JP, Karlsen K. A community-based study of sleep disorders in patients with Parkinson's disease. Mov Disord 1998;13:895-9.

3 Pal PK, Calne S, Samii A, et al. A review of normal sleep and its disturbances in Parkinson's disease. Parkinsonism and Related Disorders 1999;5:1-17.

4 Manni R, Morini R, Martignoni E, et al. Nocturnal sleep in multisystem atrophy with autonomic failure: polygraphic findings in ten patients. J Neurol 1993;240:247-50.

5 Plazzi G, Corsini R, Provini F, et al. REM sleep behavior disorders in multiple system atrophy. Neurology 1997;48:1094-7.

6 Wetter TC, Collado-Seidel V, Pollmacher T, et al. Sleep and periodic leg movement patterns in drug-free patients with Parkinson's disease and multiple system atrophy. Sleep 2000;23:361-7

7 Askenasy JJM, Weitzman ED, Yahr MD. Are periodic movements in sleep a basal ganglia dysfunction? J Neural Transm 1987;70:337-47.

8 Albin RL, Koeppe RA, Chervin RD, et al. Decreased striatal dopaminergic innervation in REM sleep behavior disorder. Neurology 2000;55:1410-12.

9 Schenck CH, Bundlie SR, Mahowald MW. Delayed emergence of a parkinsonian disorder in $38 \%$ of 29 older men initially diagnosed with rapid eye movement sleep behavior disorder. Neurology 1996;46:388-93.

10 Tison F, Wenning GK, Daniel SE, et al. The pathophysiology of parkinsonism in multiple system atrophy. Eur J Neurol 1995;2:435-44.

11 Gilman S, Low PA, Quinn N, et al. Consensus statement on the diagnosis of multiple system atrophy. J Auton Nerv Syst 1998;74:189-92.

12 ICDS (1991). International Classification of Sleep Disorders: diagnostic and coding manual. Diagnostic Classification Steering Committee (Thorpy M, Chairman). Rochester, Minn: American Sleep Disorders Association.

13 Nausieda PA, Glantz R, Weber S, et al. Psychiatric complications of levodopa therapy of Parkinson's disease. Adv Neurol 1984;40:271-7.

14 Tachibana N, Kimura K, Kitajima K, et al. REM sleep motor dysfunction in multiple system atrophy: with special emphasis on sleep talk as its early clinical manifestation. J Neurol Neurosurg Psychiatry 1997;63:678-81

15 Iranzo A, Santamaria J, Tolosa E. Continuous positive air pressure eliminates nocturnal stridor in multiple system atrophy. Lancet 2000;356: 1329-30.

16 Askenasy JJM, Yahr MD. Reversal of sleep disturbance in Parkinson's disease by antiparkinsonian therapy: a preliminary study. Neurology 1985:35:527-32

17 Andreu N, Chale JJ, Senard JM, et al. L-Dopa-induced sedation: a double-blind cross-over controlled study versus triazolam and placebo in healthy volunteers. Clin Neuropharmacol 1999;22:15-23.

18 Tandberg E, Larsen JP, Aarsland D, et al. The occurrence of depression in Parkinson's disease. A community-based study. Arch Neurol 1996;53:175-9.

19 van Hilten JJ, Weggeman $M$, van der Velde EA, et al. Sleep, excessive daytime sleepiness and fatigue in Parkinson's disease. J Neural Transm Park Dis Dement Sect 1993;5:235-44. 